\title{
Study on use of Nonvascularized Autologous Fibular Strut Graft with Autologous Cancellous Bone Graft for Treating Segmental Bone Defects of Upper Limb
}

\author{
Authors \\ Dr Kosuri Krishna Karthik, Dr B. Raviteja, Dr M. Pardhasaradhi \\ Dept of Orthopaedics, Andhra Medical College, Visakhapatnam, India
}

\begin{abstract}
Introduction: Non vascularized fibula strut graft provides a reliable means of treating pathologic conditions with segmental bone loss in developing countries where vascularized bone transfers, bone transport, allogenic bone grafts, bone graft substitutes and other such facilities or expertise may not be readily available.

Materials and Methods: Over a period of two years 12 patients with segmental bone loss either from trauma, deformity, nonunion or oncologic resection was included in the study.

Result: we observed uptake of graft, infection rate, union. Union is achieved in 8 patients. Non union in 3 patients with humeral defects and in 1 patient due to infection. All the forearm defects achieved union with a average grip strength of $82 \%$ in relation to contralateral limb.

Conclusion: Autologous free, non-vascularized fibula is a useful addition in developing countries attempting to manage segmental bone loss
\end{abstract}

Keywords: Fibular graft, Nonunion, Nerve injury.

\section{Introduction}

Segmental bone defect is a challenging situation in Orthopaedic clinical practice. ${ }^{1}$ Many surgical methods have been available for bridging those defects like: Autologous cancellous bone grafting, vascularized fibular grafting, Allograft, Bone graft substitutes and bone transport using ilizarov ring fixator.

Out of all, the vascular fibular grafting is popular because of its increased advantage in providing bone healing and fighting against infection. Bone transport using ilizarov ring fixator provided an alternative method for the reconstruction of composite skeletal and soft tissue defects however these procedures are complex and lengthy and associated with many complications.
However increasing technical expertise, difficulties in technique and availability of facilities makes the non vascular fibular strut graft with autologous cancellous bone grafting a best alternative. However the success of the procedure depends on the ability of the surrounding soft tissue to withstand the operative manipulation and ability to revascularise the transplanted graft. The purpose of the present study is to describe the experience with 12 non vascularized fibula autogenous grafts with autologous cancellous bone grafting used for reconstruction of segmental bone defects of upper limb.

\section{Materials and Methods}

There were 9 males 3 females with a mean age of 27 years (range 4-52 years). For post traumatic 
cases the time interval between the accident and fibular grafting was 4 weeks with initial management by debridement and external fixation or temporary immobilization. All the patients followed throughout the course of treatment by the surgeon who had performed the operation and follow up evaluation done by subjective and objective assessment of functional capacity and documentation of other problems associated with either reconstructed or donor limb. Radiographs were taken at the time of follow up.

Humerus bone defects were encountered in 7 cases, Radius defects encountered in 1 case and ulnar shaft defect was encountered in 1 case. The length of bone defect ranges from 7-10 cm (with the mean of $7.5 \mathrm{~cm}$ ). Bone loss because of post traumatic in 6 cases, Nonunion in 1 case, Tumour excision in 3 cases, Congenital defect in 1 case and infection in 1 case.

\section{Operative Technique}

In all the cases we used contralateral fibula as free non vascularised graft to fill the bone defect and contralateral iliac crest is used for cancellous bone harvesting. Under tourniquet control the proposed portion of fibula is exposed leaving at least $5 \mathrm{~cm}$ from the distal end. After dissection subperiosteally, the fibula was osteotomized proximally and distally with Gigli saw.

The graft length is measured about $4 \mathrm{~cm}$ longer than the bone defect. After preparing the bone ends of the gap and soft tissue bed, the medullary cavity of bone ends opened, drill holes made till

\section{Case 1}

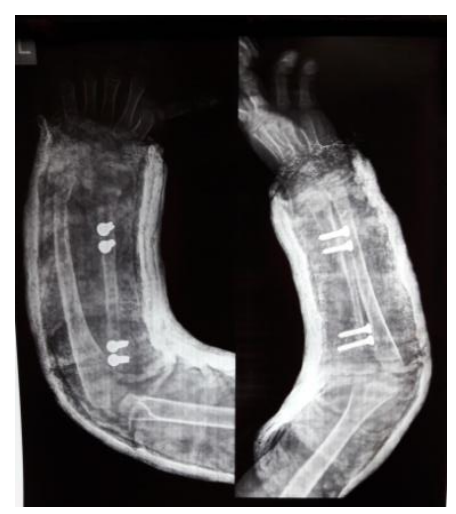

Initial X-ray bleeding and fibula Strut graft was inserted into the medullary cavity proximally and distally in 4 cases of humerus defects and in 3 cases the graft was placed in onlay method. In forearm defects graft was placed in continuity of the defect. Augmentation done with cancellous grafting and support with plate or screw provided after the procedure and immobilized in splint until the radiographic evidence of union. Cancellous grafting not done in paediatric cases.

\section{Results}

All the patients were followed for an average of 13 months (with the range of 8-24 months) after the procedure. Eight patients $(67 \%)$ return to the previous level of activity. one patient presented with symptoms of infection. within an average of 5 months all but three of the patients with humeral defect had radiographic evidence of bony Union at both proximal and distal aspect of graft bone junction.

The remaining three patients had a nonunion at the distal junction of humeral gap. The results were treated as excellent in 8 cases. None of the fibular graft gets fractured and one patient had radiographic evidence of bone resorption due to infection. No patient had pain or functional disability over the donor site. All the forearm cases were graded excellent results. Average pronation of the reconstructed forearm was 55 degrees and average supination was 40 degrees and average grip strength was about $82 \%$ of the contralateral side.

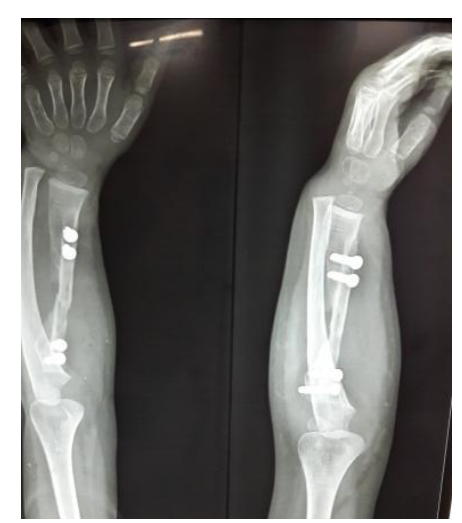

6 Weeks Follow up 
Case 2

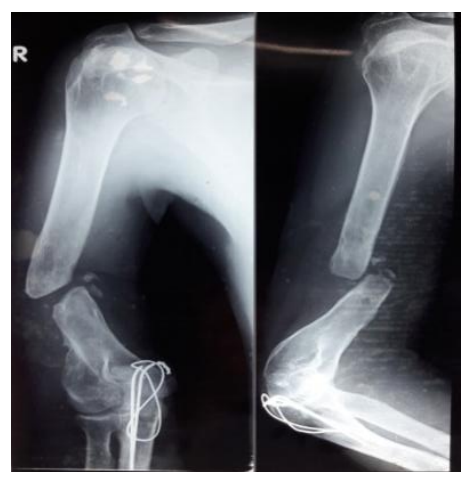

Pre op X-ray

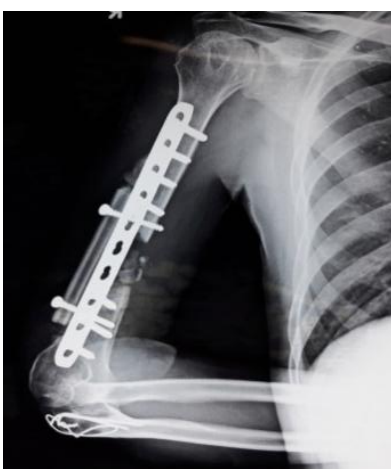

Immediade Post op

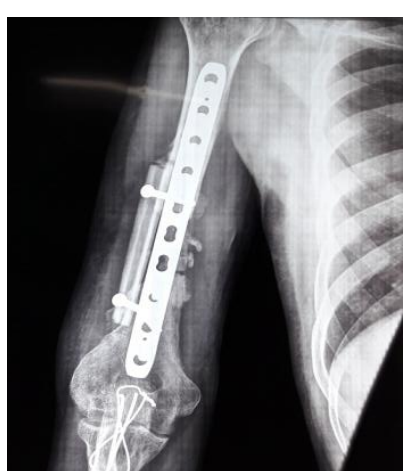

8 Weeks Follow up

\section{Discussion}

Vascular graft in combination with cancellous bone grafting provide probably the best means of dealing with Post traumatic bone defects or those created after removal of tumour. However In our present environment there is a lack of technical expertise and facilities available for these procedures.

Cancellous graft has ${ }^{2}$ osteogenic and osteoinductive properties but it is not osteoconductive, without ${ }^{3}$ desirable features of a bone graft. since it is autologous it doesn't allow transmission of virus or bacterial pathogens.

However the main drawback of cancellous bone grafting is limited number of harvesting sites and volume as well as ${ }^{4}$ high failure rate when it is used alone with donor site morbidity. Hence the combination of autologous non vascular fibular graft which is osteoconductive in combination with autologous cancellous graft gives good results. In our series the main complication was infection in one patient and non Union in three patients.

Graft was incorporated in $67 \%$ of patients treated. It is important to preserve proximal and distal $5 \mathrm{~cm}$ of fibula during the procedure to prevent common peroneal nerve injury and to retain the stability of ankle joint ${ }^{5,6}$. it is noteworthy that none of our patient had stress fracture which is common when ${ }^{7}$ greater or equal to $12 \mathrm{~cm}$ long graft was used.

\section{Conclusion}

From the findings of above series it can be drawn that autologous free non vascularised fibula and cancellous graft which are used in combination are useful addition to the management of segmental bone defect in developing countries because of a trauma or excision of tumour.

\section{References}

1. Taylor GI, Milller GD, Ham FJ. The free vascularised bone:A clinical extension of microvascular techniques. Plast Reconstr Surg 1975;55:533-44.

2. Finkemier CG. Bone grafting and bone graft substitutes.J Bone Joint Surg Am 2002;84-A:454-64.

3. Dell PC, Burchardt H, Glowsczewski FP Jr. Aroentegenographic, biomechanical and histological evaluation of vascularised and non-vascularised segmental fibula canine autografts. J bone Joint SurgAm 1985;67-A:105-12.

4. Enneking WF, Gearen PF. Fibrous dysplasia of the femoralneck: Treatment by cortical bone grafting. J Bone Joint Surg Am 1986;68:1415-22.

5. George B, Abudu A, Grimer RJ, Carter $\mathrm{SR}$, Tilman RM. The treatment of benign lesions of the proximal femur with nonvascularised autologous fibular strut grafts. J Bone Joint Surg Br 2008;90:648-51.

6. Springfield D. Autograft reconstructions. Orthop Clin North Am 1996;27:483-92.

7. De Boer HH, Wood MB. Bone changes in the vascularised fibula graft. J Bone Joint Surg Br 1989;71B:374-8. 